\title{
Differential effects of IL2R $\alpha$ and IL15R $\alpha$ over the stability of the common beta-gamma signaling subunits of the IL2 and IL15 receptors
}

Luis F. Ponce ${ }^{1,2 *}$, Galia Montalvo ${ }^{1}$, Kalet Leon ${ }^{1}$ and Pedro A. Valiente ${ }^{3+*}$

${ }^{1}$ Molecular System Biology department, Center of Molecular Immunology, Havana, Havana 11600, Cuba

${ }^{2}$ Center for Molecular Simulations, Biological Science Department, University of Calgary, Calgary, Alberta, Canada, T2N 1N4

${ }^{3}$ Center for Protein Studies, Faculty of Biology, University of Havana, Havana, Havana 10400, Cuba

*To whom correspondence: luis.poncealvarez@ucalgary.ca, and pedro.valiente79@gmail.com

$+\underline{\text { Current address: }}$ Donnelly Centre for Cellular and Biomolecular Research, University of Toronto, Toronto, Ontario, Canada, M5S 3E1 

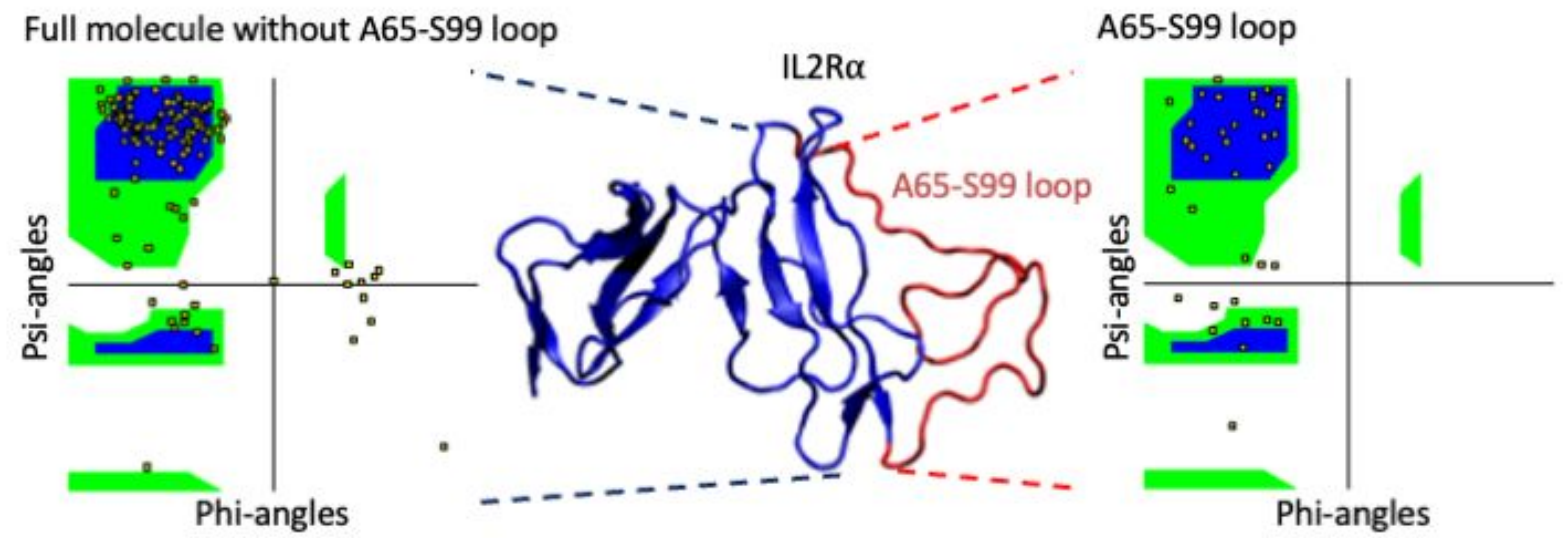

Figure S1. Structural assessment of the generated A65-S99 loop in the IL2R $\alpha$. In the center is represented the structure of the IL2R $\alpha$ chain with the reconstructed A65-S99 loop in red. In the left panel is represented the Ramachandran plot of IL2Ra without loop A65-S99 while the right panel showed the predicted loop.
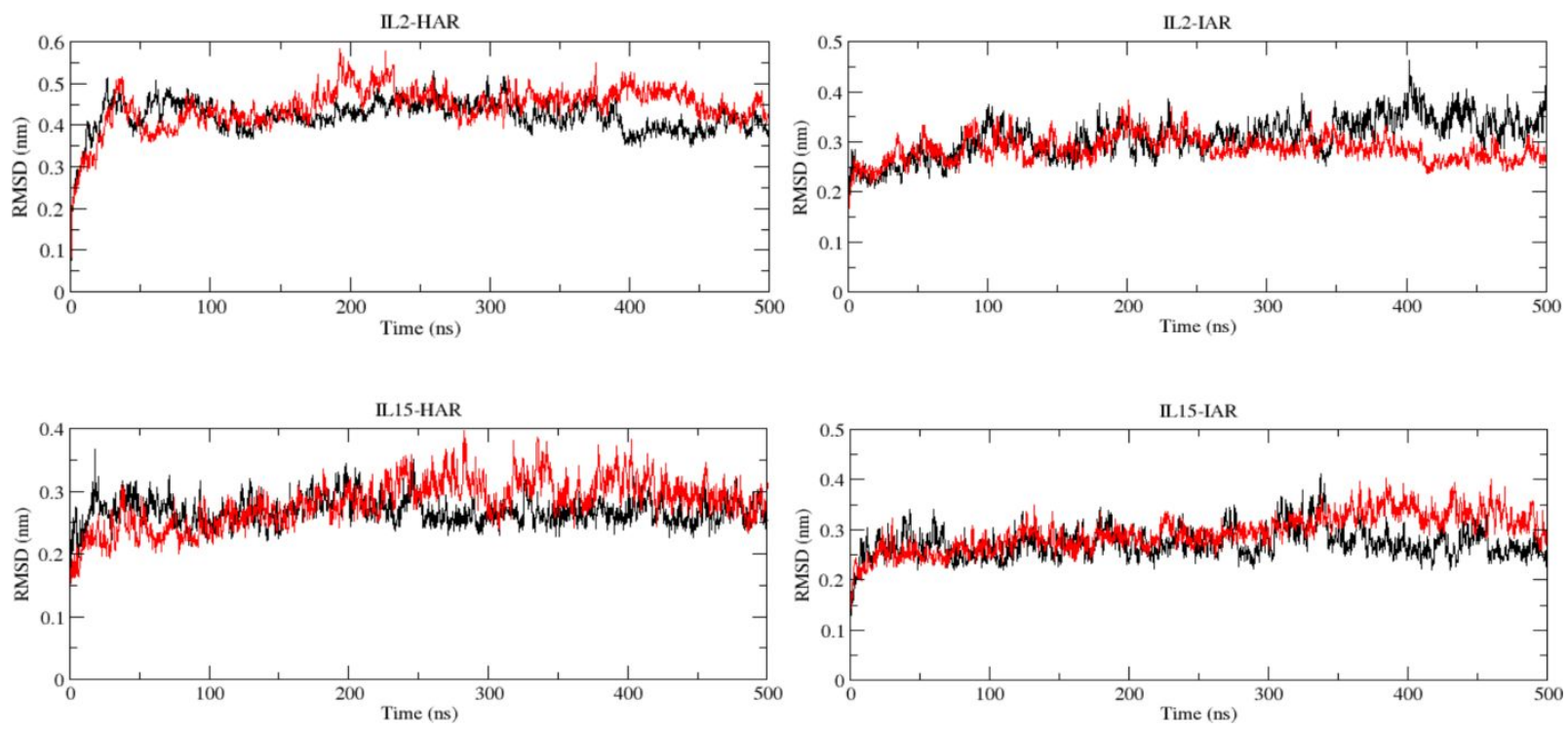

Figure S2. RMSD of the heavy atoms of the IL-receptor complexes along the MD simulation. Colors red and black correspond to different replicas of each system simulation. On the top of each graph is labeled the corresponding system. 


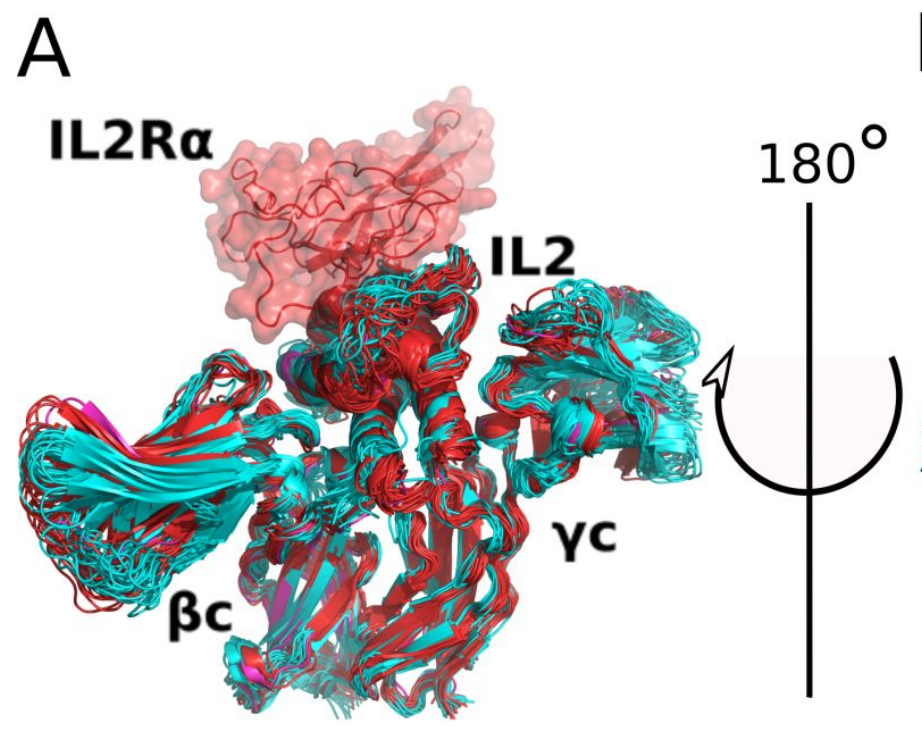

B

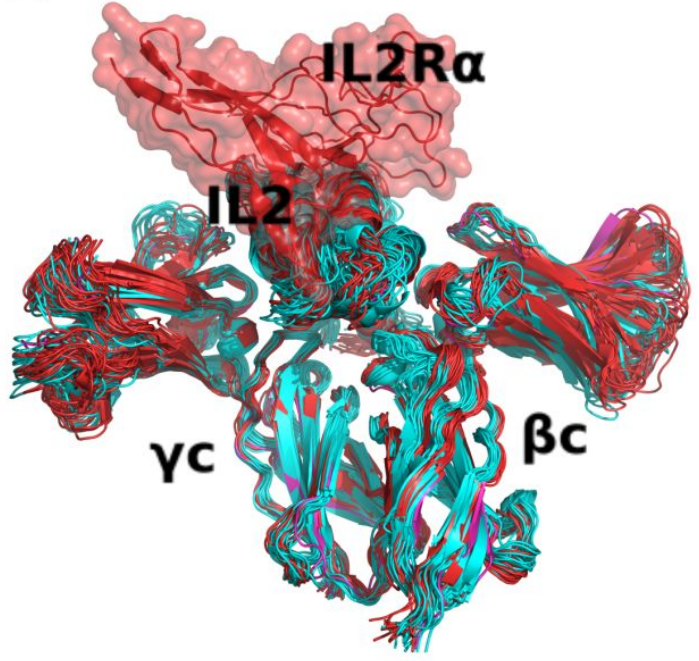

C
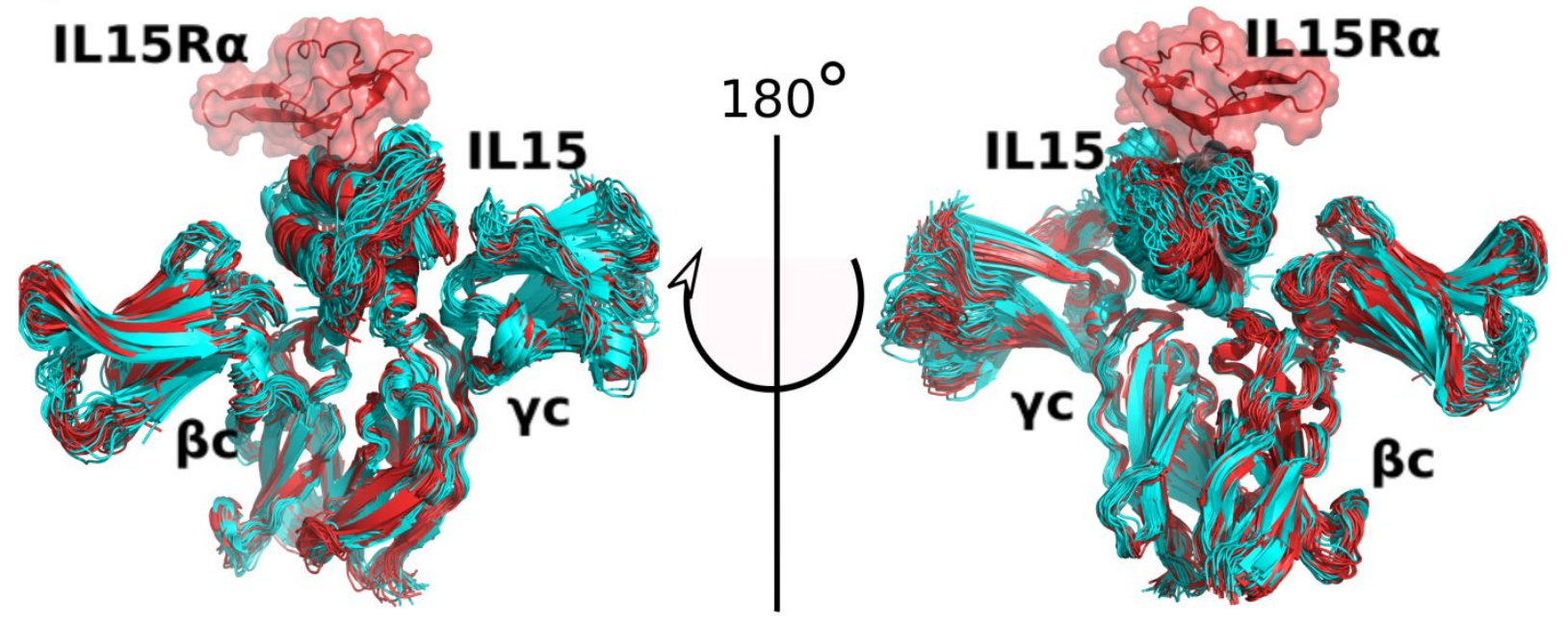

Figure S3. Comparison of the structural alignment of IL2- $\beta-\mathrm{y}_{c}$ and IL15- $\beta-\mathrm{Y}_{c}$ in the HAR and IAR complexes. A and B) Two different views of the comparison of the ensemble of structures of IL2- $\beta-\gamma_{c}$ in the HAR (in red) or IAR complex (in cyan). C and D) Two different views of the comparison of the ensemble of structures of IL15- $\beta-\gamma_{c}$ in the HAR (in red) or IAR complex (in cyan). Structures correspond to the frames in the last 400 ns MD simulations of each complex separated by $20 \mathrm{~ns}$. Red surfaces on the top indicate the region for the binding of the a chains. 


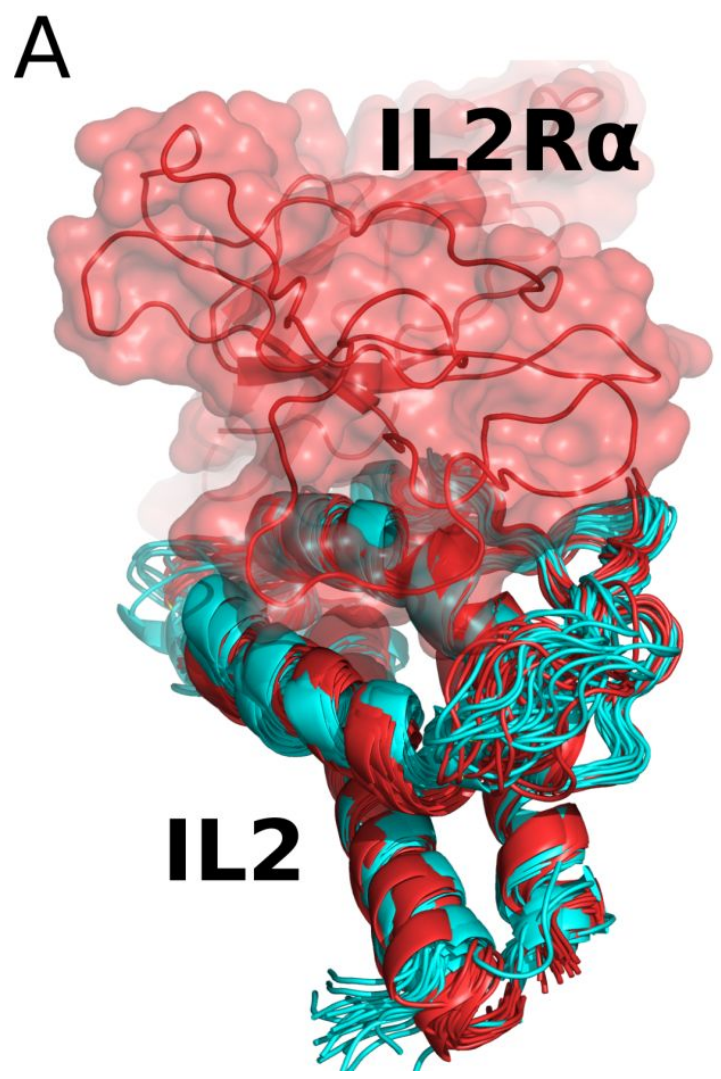

B

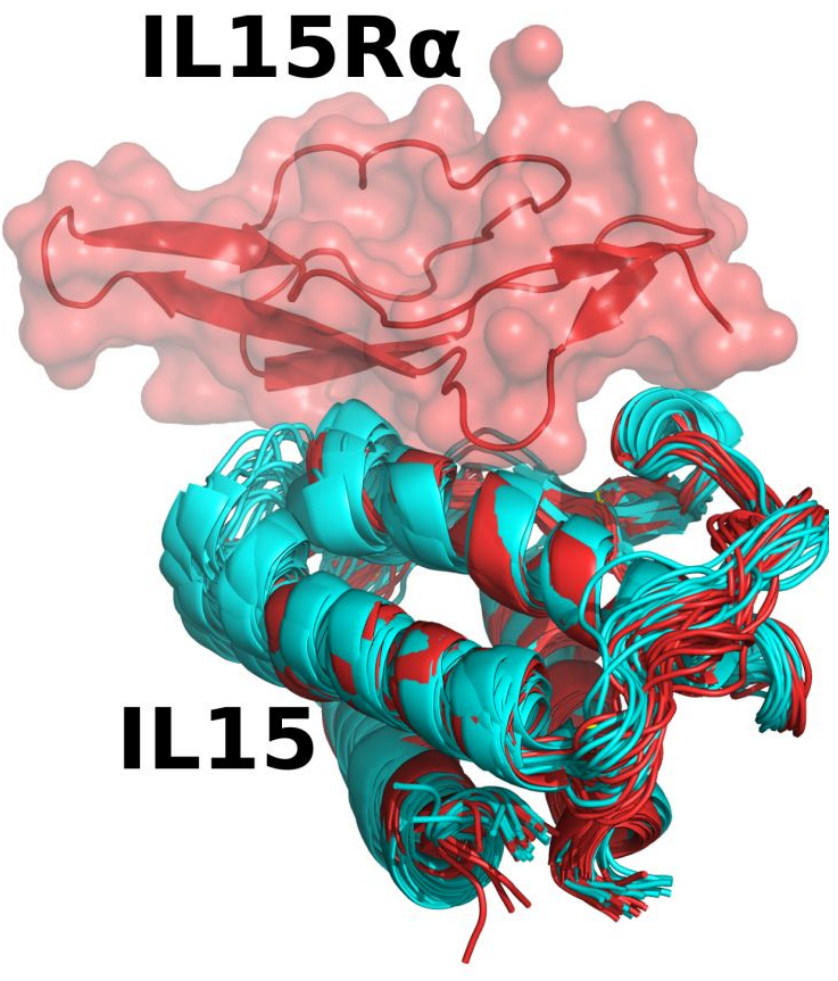

Figure S4. Minor changes in the structure of the ligands in the HAR and IAR complexes. A) comparison of the IL2 alignment between structures from the last $400 \mathrm{~ns}$ MD simulation of the HAR (in red) and IAR (in IAR) complexes. B) comparison of the IL15 alignment between structures from the last 400 ns MD simulation of the HAR (in red) and IAR (in IAR) complexes. Red surfaces on the top indicate the region for the binding of the $\alpha$ chains. 